\title{
In vitro study of retention of paired press-stud overdenture component attachments
}

\begin{abstract}
Statement of problem: Loss of retention of implant overdenture stud attachment assemblies presents maintenance problems to both the patient and dentist.

Purpose: The purpose of this in vitro study was to investigate effects of repeated separation and reunion cycles on retention of paired overdenture stud attachment assemblies set in acrylic resin.

Material and methods: Ten pairs of stud attachments were set vertically and parallel to each other (Group 1). Ten other pairs of stud attachments were set with one vertical and the other at a vertical angle of $15^{\circ}$, so that it converged on the first implant (Group 2). Baseline measurements of separation force were made for each of the ten paired attachment assemblies in the two groups. Each assembly was first subjected to a cycle of 100 separations and reunions, followed by measurement of separation force. Then, additional cycles were carried out for a total of 1000 separations and reunions, with measurement of separation force and retightening after each cycle. Repeated-values ANOVA was employed to compare the two groups at the different time intervals, with $\mathrm{p}<.05$ representing statistical significance.
\end{abstract}

Results: While significant $(\mathrm{p}<.0001)$ decay in the resistance to separation was observed for both the parallel and converging groups of paired stud attachment assemblies, these decreases were judged to have little clinical importance.

Conclusion: There was no clinically meaningful difference in retention between the first group of two parallel stud attachments and the second group with one attachment converging at an angle of $15^{\circ}$. Group 1 showed greater retention than Group 2 after the retightening adjustments.
Volume 6 Issue 5 - 2017

\author{
Ahmed AA Fathalah,' Declan Byrne, ${ }^{2}$ Noel \\ Claffey, ${ }^{2}$ Frank Houston, ${ }^{2}$ Osama Omer, ${ }^{4}$ \\ William A Brantley ${ }^{5}$ \\ 'Star Dental Laboratory LLC, Dublin, OH, USA \\ ${ }^{2}$ Department of Restorative Dentistry and Periodontology, \\ School of Dental Science, Europe \\ ${ }^{3}$ Faculty of Dentistry, Royal College of Surgeons in Ireland \\ (RCSI), Ireland \\ ${ }^{4}$ Department of Restorative Science and Prosthodontics, The \\ Ohio State University, USA
}

Correspondence: William A Brantley, Division of Restorative Science and Prosthodontics College of Dentistry, The Ohio State University, Columbus OH 43210, USA, Tel 614-292-0773, Fax 6I4-292-9422, Email brantley. I@osu.edu

Received: March 05, 2017 | Published: March 16, 2017

\section{Clinical implications}

For paired press-stud overdenture attachments from one manufacturer, there was no difference in retention force when the attachments were parallel or tilted (one at vertical angle of $15^{\circ}$ ), after approximately one year of simulated separating/reuniting cycles by a patient. The parallel attachments showed greater retention after periodic tightening than the tilted attachments.

\section{Introduction}

The mandibular two-implant overdenture has become a widely used restoration for edentulous patients, and the success of this type of restoration has been documented. ${ }^{1-4}$ However, there has been a report of high failure rate of the retentive components used in these restorations, which seems to be related to their repeated. ${ }^{5}$ For example, concerns have been expressed about the effect of wear between components of the commonly used Bona-type stud attachments. ${ }^{6}$ There have also been concerns that the relative angulation of these components may affect their rate of wear..$^{7-9}$ The purpose of this in vitro study was to investigate the possible effect of repeated separation and reunion cycles on the retention of paired overdenture stud attachment assemblies set in acrylic resin. The present results are compared to those reported in other studies, ${ }^{9-14}$ providing some insight into these concerns and the major factors involved.

\section{Material and methods}

\section{Test model}

Two cylindrical blocks of acrylic resin (Vari-Set 20, Met Prep Ltd, Coventry, UK), approximately $36 \mathrm{~mm}$ in diameter, were constructed. One end on each block was cut flat and polished (Met Prep PH Machine). For the first set (Group 1) of 10 test specimens, two analogues for stud attachments (ITI ${ }^{\circledR}$ Straumann) were secured vertically in one acrylic block, $22 \mathrm{~mm}$ from the center and along a diameter line, with the aid of a surveyor (Bachman ${ }^{\circledR}$ Parallelometer, Cendres \& Metaux, Switzerland), as shown in Figure 1. A second set (Group 2) of 10 specimens was assembled in the same manner, except one attachment was set vertically and the second was set at a $15^{\circ}$ angle toward it, as shown in Figure 2 . The attachments for the stud assemblies of both Groups 1 and 2 were secured to the other acrylic block, using the same material. A removable square metal rod was fastened to the second acrylic block and fit into corresponding 
square tubing in the first block, serving as a guidance rod for seating during the separation and reunion cycling. A nut was incorporated in the upper acrylic block to facilitate the placement of a mandrel. Each specimen was placed in a universal testing machine (H5K-S, Tinius Olsen), the guidance rod removed, and the separating force recorded after successive 100 separating/uniting cycles up to a total of 1000 cycles. The crosshead speed for the separating/uniting cycles was set to simulate the removal and insertion of an overdenture by a patient. After 1000 cycles, the female components for the stud attachments in both groups were tightened (adjusted) by a single operator, using the tool supplied by the manufacturer. Then the 10 sets of 100 separating/ uniting cycles, with separating force measurements, were repeated.

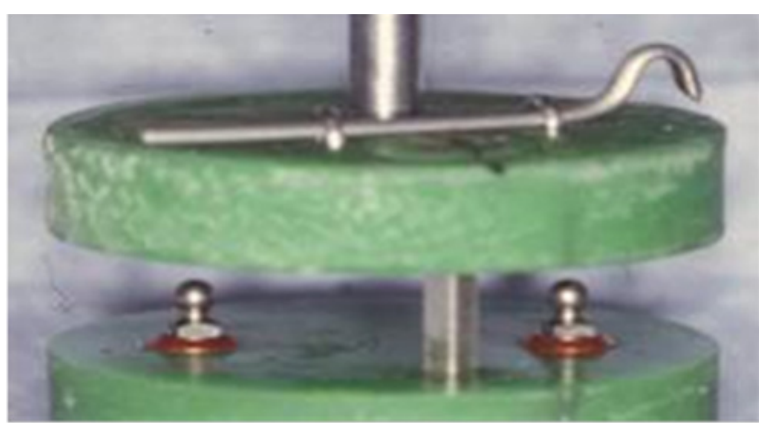

Figure I Group I specimen with pair of stud attachments set vertically and parallel.

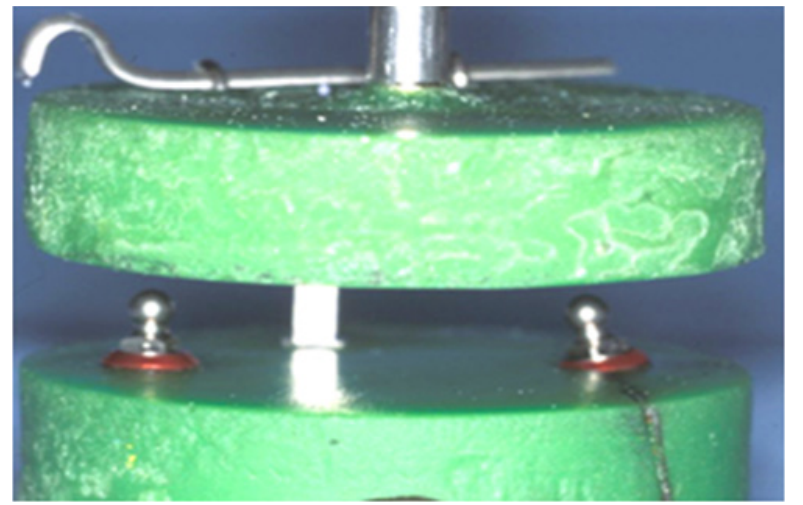

Figure 2 Group 2 specimen with one attachment set vertically and second set at $15^{\circ}$.

\section{Data analysis}

A repeated-measures ANOVA model was employed, with Group (1 and 2) as the first factor, the 10 repeated measurements after successive 100 separating/uniting cycles as the second factor, and condition (nottightened or tightened) as the third factor. The individual specimens within each group were regarded as a nested variable. This statistical model examined the main effects for values of separation force as follows:

A. Group (the two groups of test specimens with parallel and tilted press-stud overdenture attachments).

B. Measurement over Time (after each of the successive 100 cycles for the two groups).

C. Condition (before adjustment and after adjustment). The interaction between Group and Condition was also examined.

\section{Results}

The results for both alignment groups (parallel and tilted) of the stud attachment analogues, and for the before-adjustment (tightening) and after-adjustment conditions, are summarized in Table 1 and presented graphically in Figure 3. The codes $\mathrm{M}_{1}-\mathrm{M}_{10}$ in Table 1 indicate the mean measured values of retention force $(\mathrm{N})$ for the 10 specimens in each group after each period of 100 separating/uniting cycles. Both the parallel and tilted specimen groups show higher retention force values after tightening than before tightening. After the tightening adjustments, the group with parallel press-stud attachments displayed consistently higher retention force values than the group with tilted press-stud attachments, whereas both groups consistently showed equivalent values before tightening. The repeated-measurement ANOVA revealed that significant $(\mathrm{p}=.0043)$ overall differences were found for the experimental model, with both Group and Specimen being significant $(p \leq .0001)$. Significant differences $(p \leq 0001)$ were also found for Condition (before and after adjustment). There was significant interaction between Group and Condition, but this was judged to have minimal practical importance. Significant differences $(\mathrm{p}=.0004)$ were found in the repeated retention force Measurement over Time for the successive 100 separating/uniting cycles. However, the small but relatively consistent decay in retention force values for the 2 groups, before and after tightening (Table 1) (Figure 3), was judged to have minimal practical importance. There was a significant interaction between Measurement over Time and Group $(\mathrm{p}=.02)$, but again this was judged to have minimal practical importance. There was no significant interaction between Measurement over Time and Condition (before and after adjustment). The principal contribution to the significant difference between Groups 1 and 2 resulted from the differences in retention force following the tightening adjustments.

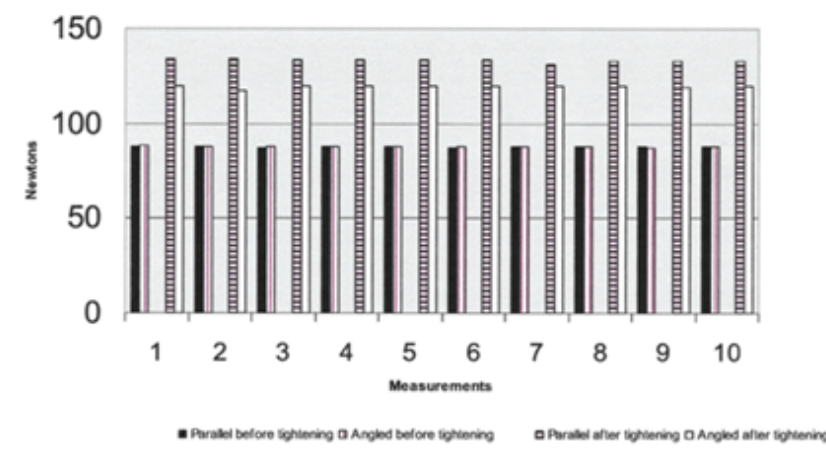

Figure 3 Retention force results for Groups I and 2, before and after tightening.

\section{Discussion}

Figure 3 \& Table 1 show that there was minimal difference in the mean retention force for Group 1 (parallel aligned attachments) and Group 2 (tilted attachments) for the before-adjustment (tightening) condition after each 100 separating/uniting cycles. After adjustment (tightening), both Groups 1 and 2 displayed a marked increase in retention force, which was greater for Group 1. (The tightening adjustment after each group of 100 cycles is a unique feature of the present study.) This difference in retention force was attributed to the increased contact between the two stud components for Group 1 , in which the female component contacted the greatest diameter of 
the male component. In contrast, for Group 2 the female component of the angled attachment only contacted one point of the greatest diameter of the male attachment and the second point above this greatest diameter. While plausible, verification of this conjecture would require scanning electron microscope examination of the male and female surfaces for evidence of contact wear. Using Straumann spherical ball abutments hand-tightened onto ITI dental implants, with gold and titanium matrices coupled to these abutments, Gulizio et al. ${ }^{9}$ observed significant differences in retention of the gold matrices when the ball abutments were positioned at vertical angles of $20^{\circ}$ and $30^{\circ}$, but not at angles of $10^{\circ}$ and $0^{\circ}$. The positioning angle from the vertical axis was not a significant factor for retention of the titanium matrices. The effect of large numbers of uniting/separating cycles on dislodging force was not investigated by Gulizio et al. ${ }^{9}$ The 1000 separating/uniting cycles in the present study corresponds to a patient removing the overdenture three times daily for a period of approximately one year. Fromentin et al. ${ }^{10}$ employed a testing methodology in which Straumann spherical stud abutments, having both vertical and $20^{\circ}$ alignments, were removed from a retentive gold housing over a testing period of 10,000 continuous cycles. While a large increase in the seating/unseating force were observed during the first 300 cycles, no significant difference was found between the initial and final values of seating/unseating force, suggesting that use of substantially more than the 1000 testing cycles would not have altered the results of the present study. Although one limitation of this study was its in vitro nature, with ambient conditions and the absence of a lubricant, this protocol has also been followed in some recent studies $^{10-14}$ of overdenture retention. Other recent investigations have employed artificial saliva at room temperature ${ }^{11}$ or water at $37^{\circ} \mathrm{C}^{12}$ for the testing environments. To date no investigation of overdenture retention after a large number of separating/reuniting cycles has fully compared the effects of testing temperature (ambient or intraoral) and testing environment (dry vs. wet: water or artificial saliva). Another limitation of the present study was that the appliances were subjected to a fixed path of placement and removal, without being subjected to the compressive forces that may also arise under clinical conditions of overdenture insertion/removal by a patient. Future research should compare overdenture attachments from several manufacturers, have a range of vertical inclinations of the paired attachments from $0^{\circ}$ to $30^{\circ}$, employ an instrumented tool that enables a constant tightening torque when making adjustments, utilize a larger number of uniting/ separating cycles simulating up to 5 years of patient use, and compare the use of dry and wet testing environments. Scanning electron microscope observations of the wear of the male and female stud components, similar to those performed by Abi Nader et al. ${ }^{13}$ are recommended, complemented by strain energy measurements in the manner of Petropoulos and Mante,,${ }^{14}$ at periodic intervals during the uniting/separation cycles. One would anticipate that the changes in strain energy would be correlated with the amount of wear.

Table I Mean retention forces $(\mathrm{N})$ for Groups I (parallel) and 2 (angled) and before-tightening adjustment (B) and after-tightening (A) conditions. Group measurements $M_{1}-M_{10}$ were made after successive 100 separating/uniting cycles

\begin{tabular}{lllllllllll} 
Group/condition & MI & M2 & M3 & M4 & M5 & M6 & M7 & M8 & M9 & MI0 \\
\hline I/Before & 88.14 & 87.78 & 87.35 & 87.89 & 87.68 & 87.46 & 87.79 & 87.82 & 87.8 & 87.87 \\
I/After & 133.97 & 133.88 & 133.64 & 133.76 & 133.42 & 133.4 & 131.25 & 132.9 & 133.27 & 133.22 \\
2/Before & 88.4 & 87.88 & 87.93 & 87.89 & 87.86 & 87.68 & 87.96 & 87.95 & 87.52 & 87.85 \\
2/After & 120.11 & 117.62 & 119.86 & 119.92 & 119.71 & 119.7 & 119.77 & 120 & 119.51 & 119.67 \\
\hline
\end{tabular}

\section{Conclusion}

Under the conditions of this in vitro study, the following conclusions can be drawn:

1. No appreciable difference in retention force after 1000 separating/uniting cycles was found between Group 1 with the two parallel press-stud overdenture attachments and Group 2 with one attachment converging at an angle of $15^{\circ}$.

2. Group 1 showed greater retention force after the tightening adjustment had taken place following successive 100 separating/ uniting cycles.

\section{Funding}

None.

\section{Acknowledgements}

None.

\section{Conflicts of interest}

The authors declare that there is no conflict of interest.

\section{References}

1. Krennmair G, Ulm C. The symphyseal single-tooth implant for anchorage of a mandibular complete denture in geriatric patients: a clinical report. Int J Oral Maxillofac Implants. 2001;16(1):98-104.

2. Meijer HJA, Raghoebar GM, Van't Hof MA, et al. Implant-retained mandibular overdentures compared with complete dentures; a 5-years' follow-up study of clinical aspects and patient satisfaction. Clin Oral Implants Res. 1999;10(3):238-244.

3. Naert I, Gizani S, Vuylsteke M, et al. A 5-year prospective randomised clinical trial on the influence of splinted and unsplinted oral implants retaining a mandibular overdenture: prosthetic aspects and patient satisfaction. J Oral Rehabil. 1999;26(3):195-202.

4. Raghoebar GM, Meijer HJA, Stegenga B, et al. Effectiveness of three treatment modalities for the edentulous mandible. A five-year randomized clinical trial. Clin Oral Implants Res. 2000;11(3):195-201.

5. Goodacre CJ, Bernal G, Rungcharassaeng K, et al. Clinical complications with implants and implant prostheses. J Prosthet Dent. 2003;90(2):121132.

6. Gotfredsen K, Holm B, Sewerin I, et al. Marginal tissue response adjacent to Astra Dental Implants supporting overdentures in the mandible. A 2-year follow-up study. Clin Oral Implants Res. 1993;4(2):83-89. 
7. Walton JN, Huizinga SC, Peck CC. Implant Angulation: A measurement technique, implant overdenture maintenance, and the influence of surgical experience. Int J Prosthodont. 2001;14(6):523-530.

8. Mericske Stern R. Forces on implants supporting overdentures: a preliminary study of morphologic and cephalometric considerations. Int J Oral Maxillofac Implants. 1993;8:254-263.

9. Gulizio MP, Agar JR, Kelly JR, et al. Effect of implant angulation upon retention of overdenture attachments. J Prosthodont. 2005;14(1):3-11.

10. Fromentin O, Lassauzay C, Abi Nader S, et al. Testing the retention of attachments for implant overdentures-validation of an original force measurement system. J Oral Rehabil. 2010;37(1):54-62.
11. Fakhry A, Tan SC, Heiner AD, et al. Methodology for measuring the in vitro seating and unseating forces of prefabricated attachment systems used to retain implant overdentures. J Prosthodont. 2010;19(2):87-94.

12. Chung KH, Whiting D, Kronstrom M, et al. Retentive characteristics of overdenture attachments during repeated dislodging and cyclic loading. Int J Prosthodont. 2011;24(2):127-129.

13. Abi Nader S, De Souza RF, Fortin D, et al. Effect of simulated masticatory loading on the retention of stud attachments for implant overdentures. $J$ Oral Rehabil. 2011;38(3):157-164.

14. Petropoulos VC, Mante FK. Comparison of retention and strain energies of stud attachments for implant overdentures. J Prosthodont. 2011;20(4):286-293. 